\title{
Interaction of a Cylindrical Tank Bottom with an Elastic Foundation
}

\author{
Andrey Leontyev ${ }^{*}$, Avgustina Astakhova and Mikhail Maksimov \\ Moscow State University of Civil Engineering, Yaroslavskoe shosse, 26, Moscow, 129337, Russia
}

\begin{abstract}
The problem of static analysis of a circular cylindrical shell located on the elastic base of the Winkler and reinforced by longitudinal ribs is considered. The stiffeners have a rectangular cross section. The external action is represented by forces evenly distributed along the longitudinal axis, acting on the ribs from the upper structure. It is assumed that at the ends of the shell there are flat vertical walls, which give the contour absolute rigidity in the transverse direction and do not interfere with the longitudinal movement of the shell points. The General moment theory of a circular cylindrical shell is used to solve the problem. A computer program has been developed to implement the proposed algorithm. With the help of the program, a number of examples of calculation are made In the given examples, the influence of such factors as the relative length and thickness, the slope angle of the solution shell, the relative stiffness of the onboard elements on the stress state of the shell is analyzed.
\end{abstract}

\section{Introduction}

We consider a circular cylindrical shell of length $l$, located on an elastic base and reinforced along the longitudinal edges of the ribs of rectangular cross-section (figure 1).

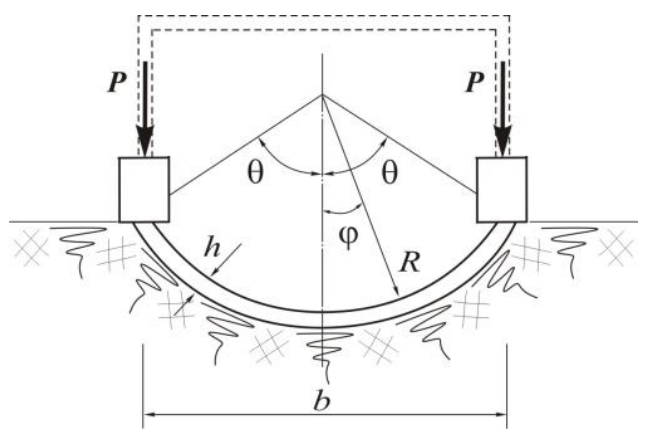

Fig. 1. Problem setting

\footnotetext{
* Corresponding author: an_leontiev@mail.ru
} 
We assume that the external action is represented by forces $P$ uniformly distributed along the longitudinal axis acting on the edges from the upper structure shown in figure 1 with a dotted line. Another assumption is that flat vertical walls are at the flanks of the shell, which render the shell's outline infinitely stiff in the transverse direction and do not impede longitudinal displacement of the shell's points.

\section{State of the problem}

To determine the elastic base reactions, assume that the shell is immersed in the elastic medium as a rigid stamp by the value of $A$ (figure 2). Besides, Winkler's hypothesis gives rise to the conclusion that the shell will be exposed to the normal $q$ and tangential $r$ response:

$$
q=k w=A k \cos \varphi, \quad r=k_{s} v=A k_{s} \sin \varphi .
$$

Here $w$ and $v$ are the normal and tangential displacements of the base surface points, $k$ and $k_{s}$ - subgrade ratios corresponding to these displacements, the last of which takes into account the friction forces arising between the shell and the base.

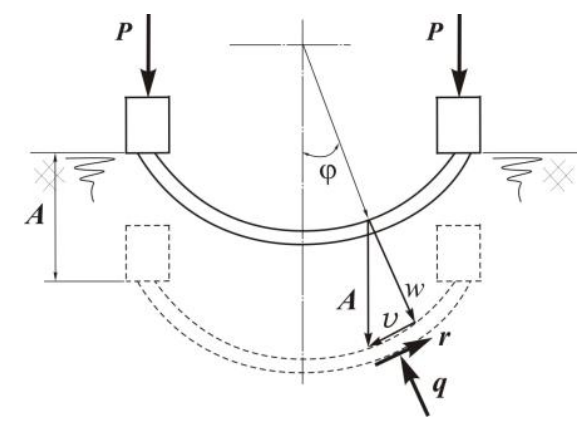

Fig. 2. Shell displacement and reactions of the base

\section{Analytical method of solving}

The vertical displacement $A$ is determined based on the equilibrium condition which makes it possible to obtain the expression below:

$$
A=\frac{2 P}{R\left[(\theta+\sin \theta \cos \theta) k+(\theta-\sin \theta \cos \theta) k_{s}\right]} .
$$

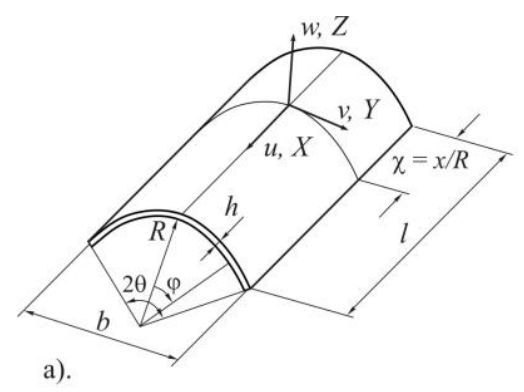

b).

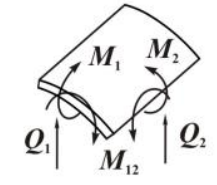

c).

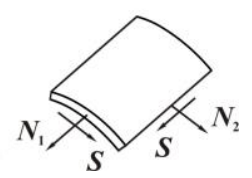

Fig. 3. Parameters and internal forces of the cylindrical shell 
The General moment theory of a circular cylindrical shell (figure 3) is used to solve the problem. [1].

The above boundary conditions specified at the ends allow us to decompose all the required quantities and loads into single trigonometric series along the longitudinal coordinate $x$. E.g., displacements of the shell can be presented as follows:

$u(x, \varphi)=\sum_{n} U_{n}(\varphi) \cos \lambda_{n} \frac{x}{R}, \quad v(x, \varphi)=\sum_{n} V_{n}(\varphi) \sin \lambda_{n} \frac{x}{R}, \quad w(x, \varphi)=\sum_{n} W_{n}(\varphi) \sin \lambda_{n} \frac{x}{R}$,

where $\lambda_{n}=\frac{n \pi R}{l}$.

When solving the problem in displacements and in the absence of load $(X=Y=Z=0)$, the system of three differential equations of equilibrium with respect to the functions $U_{n}, V_{n}$, $W_{n}[2]$ is reduced to one ordinary differential equation of the eighth order:

$$
W_{n}^{V I I I}-4 \lambda_{n}^{2} W_{n}^{V I}+6 \lambda_{n}^{4} W_{n}^{I V}-4 \lambda_{n}^{6} W_{n}^{I I}+\lambda_{n}^{4}\left(\lambda_{n}^{4}+\frac{1}{c^{2}}\right) W_{n}=0
$$

where $\quad c^{2}=\frac{h^{2}}{12 R^{2}}$.

The general integral of this homogenous equation $W_{n}$ is formulated via hyperbolictrigonometric functions with an accuracy of eight integration constants. The same constants are part of the displacement expressions $U_{n}$ and $V_{n}$, which are determined via the deflection function $W_{n}$.

In the presence of an external load to the general integrals $W_{n}, U_{n}, V_{n}$ are added partial integrals $W_{n}^{0}, U_{n}^{0}, V_{n}^{0}$ which in the case of reactive load (1) can be represented as

$$
W_{n}^{0}=A_{n} \cos \varphi, \quad U_{n}^{0}=B_{n} \cos \varphi, \quad V_{n}^{0}=C_{n} \sin \varphi .
$$

Here

$$
\begin{aligned}
& A_{n}=-\frac{1}{\lambda_{n}^{4}}\left[\left(1+\lambda_{n}^{2}\right)^{2} Z_{n}+\left(1+2 \lambda_{n}^{2}\right) Y_{n}\right], \quad B_{n}=\frac{1}{\lambda_{n}^{3}}\left(Z_{n}+Y_{n}\right), \\
& C_{n}=\frac{1}{\lambda_{n}^{4}}\left(1+\lambda_{n}^{2}\right)\left(Z_{n}+Y_{n}\right), \quad Y_{n}=\frac{4 A R^{2}}{n \pi E h} k_{s}, \quad Z_{n}=\frac{4 A R^{2}}{n \pi E h} k,
\end{aligned}
$$

where $E$ - the elasticity modulus of the shell material.

The general solution of the problem is written, thus, in the form of

$$
\bar{W}_{n}=W_{n}+W_{n}^{0}, \quad \bar{U}_{n}=U_{n}+U_{n}^{0}, \quad \bar{V}_{n}=V_{n}+V_{n}^{0} .
$$

To determine the integration constants at each of the longitudinal edges of the shell, four boundary conditions can be formulated.

In the case of support of the longitudinal edge of the shell on a beam of rectangular cross-section (figure 4,a) we assume that the connection with the beam completely eliminates longitudinal displacements $U_{n}$ of the shell's edge. In this case, based on the conditions of continuity of displacement of the edge of the shell and beam, you can write the following four boundary conditions:

$$
\bar{W}_{n}^{\text {ver }}=W_{n}^{b}, \quad \bar{V}_{n}^{\text {hor }}=V_{n}^{b}, \quad U_{n}=0, \quad \gamma_{n}=\psi_{n},
$$

where $\bar{W}_{n}^{\text {ver }}, \bar{V}_{n}^{\text {hor }}$ - denote vertical and horizontal displacements of the shell's edge, $W_{n}^{b}, V_{n}^{b}$-deflections of the beam in the vertical and horizontal planes, $\psi_{n}$ - torsion angle of the beam, $\gamma_{n}=\frac{1}{R} \bar{W}_{n}^{I}$ - rotational angle of the shell's edge (figure 4,b,c). 
a).

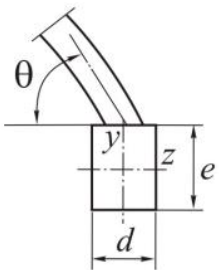

b).

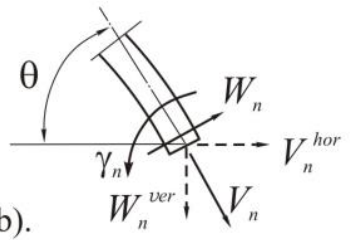

c).

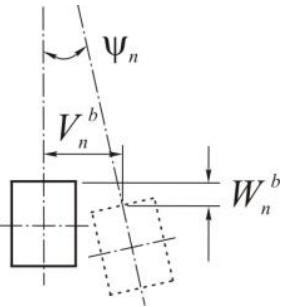

Fig.4. Interaction of the longitudinal edge of the shell with the stiffening bar

Opening conditions (7) with the help of known dependences of the resistance of materials, we obtain:

$$
\begin{aligned}
&-\bar{W}_{n} \cos \theta+\bar{V}_{n} \sin \theta=\frac{R^{4}}{\lambda_{n}^{4}\left(E I_{z}\right)_{b}}\left(Q_{2} \cos \theta-N_{2} \sin \theta-\frac{4 P}{n \pi}\right), \\
& \bar{W}_{n} \sin \theta+\bar{V}_{n} \cos \theta=-\frac{R^{4}}{\lambda_{n}^{4}\left(E I_{y}\right)_{b}}\left(Q_{2} \sin \theta+N_{2} \cos \theta\right),
\end{aligned}
$$

$\bar{U}_{n}=0, \quad M_{2}-\frac{b}{2}\left(Q_{2} \sin \theta+N_{2} \cos \theta\right)=-\left(G I_{t o r}\right)_{b} \frac{\lambda_{n}^{2}}{R^{3}} \bar{W}_{n}^{I}$,

where $I_{z}, I_{y}, I_{t o r}$ - inertia moments of the beam cross section, $G$ - modulus of shear, $M_{2}$, $Q_{2}, N_{2}$ - internals efforts in longitudinal sections of the shell (figure 3,b,c). Boundary conditions (7) take the final form after the expression in formulas (8) of displacements $\bar{U}_{n}$, $\bar{V}_{n}$ and internals efforts $M_{2}, Q_{2}, N_{2}$ through the deflection function $\bar{W}_{n}$.

After determining the integration constants and finding general solutions (6), the internals efforts are determined by the known formulas of the theory of cylindrical shells [1].

\section{Examples of calculation}

A PC software program was developed to enforce the proposed algorithm, a range of calculations were run using this software. These calculations served to analyze such factors having an effect on the stressed state of the shell as relative length and thickness, expansion angle of the shell $\theta$, relative stiffness of shell edge reinforcement etc. The output of one of the calculations is presented below.

The following input data are adopted for the shell in question. The relative length and thickness are suggested to be $l / b=2, R / h=20$ respectively, the stiffening bars are squaresectioned with the square side being $d=4 h$, the material of the shell and of the stiffening bars have the same elasticity modulus $E$. As an external action, consider a uniformly distributed $10 P$ intensity load applied along the edges of the shell.

Figures 5,a and 5,b present non-dimensional distribution diagrams of the transverse bending moments $M_{2}$ and longitudinal forces $N_{2}$, designed based on $\theta=60^{\circ}$ for two values of the subgrade reaction $k_{s}$. You can see that the account of forces of friction when $k_{s}=0,2 k$ (dotted lines), abruptly change the magnitude of the longitudinal forces $N_{2}$ and less impact on the magnitude of the bending moments $M_{2}$.

Figure 6 regards the same shell and displays the effect of the relative stiffness of an edge in torsion with $k_{s}=0,2 k$ on the amount of bending moments $M_{2}$ in the sections $\varphi=0$ (at the center of the shell) and $\varphi=\theta$ (at the edge of the shell). Dotted lines represent the shell with edges rigidly clamped. One can see that given $\left(G I_{t o r}\right)_{b} / D b>15$ the stiffness of the bar virtually corresponds to the shell edge being rigidly clamped. 
a).

b).

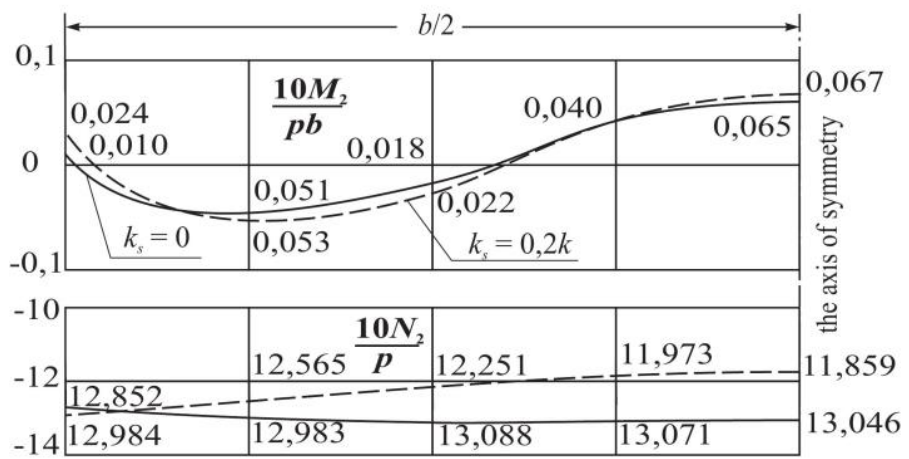

Fig. 5. Distribution diagrams of bending moments $M_{2}$ and longitudinal forces $N_{2}$

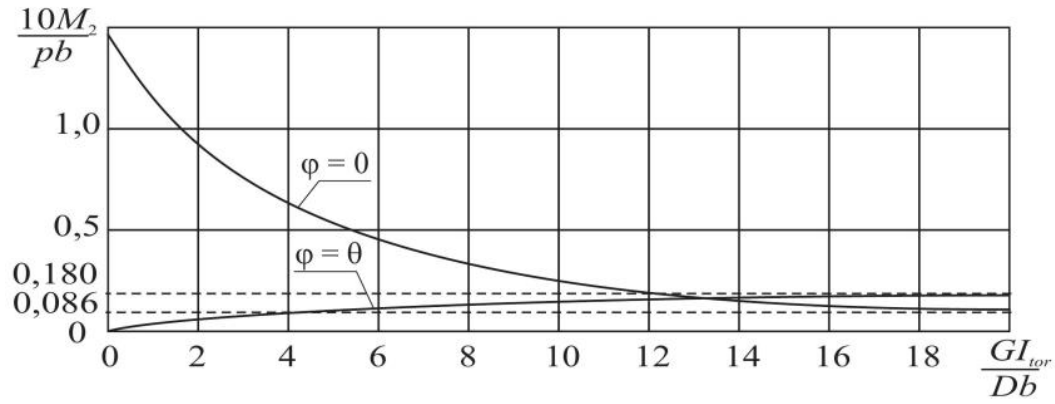

Fig. 6. Influence of the relative stiffness of the rib during torsion on the bending moments $M_{2}$

\section{Conclusions}

Given in the article the method of calculation of circular cylindrical shell located on an elastic foundation, allows determining the internal forces in the shell. It should be noted that the problems of calculation of structures on elastic foundation do not lose their relevance. The development of new models makes it possible to find engineering solutions that allow for analysis at the pre-project stage and to conduct a simpler analysis of the stress-strain state of structures and the ground base [3-6].

\section{References}

1. V.Z. Vlasov, General shell theory (Gostechizdat, 1949).

2. N.N. Leontiev, A.N. Leontiev, B.H. Monsef, Theor. and Exp. Stud. of Str. and Stif. of Struc. Elem. (MSUCE, 2001).

3. V.I. Andreev, E.V. Barmenkova, A.V. Matveeva, Proc. of the Univ. Constr., 9 (2010)

4. V.I. Andreev, E.V. Barmenkova, Struct. Mech. and Calc. of Struct., 6 (2010)

5. V.I. Andreev, E.V. Barmenkova, Appl. Mech. and Mater., 204-208 (2012)

6. V.I. Andreev, A.V. Matveeva, E.V. Barmenkova, Appl. Mech. and Mater., 351-352 (2013) 